\title{
Les réserves de la Société française de médecine d'urgence sur les recommandations 2018 de la Surviving Sepsis Campaign
}

\author{
The French Society of Emergency Medicine Expression of concern regarding the 2018 Update \\ of the Surviving Sepsis Campaign
}

\begin{abstract}
Y. Freund (Président de la commission scientifique SFMU) · P.-G. Claret (Président de la commission des référentiels SFMU) - M. Maignan (Président de la commission recherche SFMU) · K. Tazarourte (Vice-président de la SFMU) ·
\end{abstract} A. Ricard-Hibon (Présidente de la SFMU)

Reçu le 18 mars 2019; accepté le 3 avril 2019

(C) SFMU et Lavoisier SAS 2019

En 2018, la Surviving Sepsis Campaign (SSC) a révisé son protocole de prise en charge (sepsis bundle) [1]. Ce protocole de prise en charge recommandait en 2016 de prélever

Y. Freund $(\bowtie) \cdot$ P.-G. Claret $\cdot$ M. Maignan $\cdot$ K. Tazarourte A. Ricard-Hibon

Société française de médecine d'urgence,

103, boulevard Magenta, F-75010 Paris

e-mail : yonathan.freund@aphp.fr

\section{Y. Freund}

Service d'accueil des urgences, groupe hospitalier Pitié-Salpêtrière,

Assistance publique-Hôpitaux de Paris,

47-83, boulevard de 1'Hôpital, F-75013 Paris, France

Faculté de médecine, Sorbonne Université,

91, boulevard de 1'Hôpital, F-75013 Paris, France

P.-G. Claret

Pôle anesthésie-réanimation-douleur urgences, centre hospitalier universitaire de Nîmes,

4, rue du Professeur-Robert-Debré, F-30029 Nîmes, France

M. Maignan

Urgences Samu-Smur, CHU de Grenoble-Alpes,

F-38000 Grenoble, France

Inserm U1042, laboratoire hypoxie-physiopathologie, université de Grenoble-Alpes, F-38000 Grenoble, France

\section{K. Tazarourte}

Pôle urgence, Samu 69, groupe hospitalier Édouard-Herriot, hospices civils de Lyon, 5, place d'Arsonval,

F-69008 Lyon, France

Université Claude-Bernard-Lyon-I, F-69008 Lyon, France

\section{A. Ricard-Hibon}

Samu Centre 15 du Val-d'Oise, Smur Pontoise, centre hospitalier René-Dubos, 6, avenue de l'Île-de-France, CS 90079 Pontoise, France des hémocultures, le lactate, et de débuter une antibiothérapie à large spectre associée à l'administration de $30 \mathrm{ml} / \mathrm{kg}$ de soluté cristalloïde, et ce, dans les trois heures suivant le diagnostic de sepsis [2]. Bien que fondées sur de faibles niveaux de preuve, ces recommandations s'appuyaient sur plusieurs études importantes, rétrospectives ou prospectives - mais observationnelles, qui soulignaient le lien entre respect de ces recommandations et amélioration du pronostic [3-5]. Les nouvelles recommandations de 2018 recommandent désormais de réaliser le protocole de prise en charge en une heure après le triage au lieu de trois heures. À l'instar d'autres sociétés scientifiques (Society for Critical Care Medicine, Infectious Disease Society of America, American College of Emergency Physicians) et en particulier de l'European Society of Emergency Medicine (EUSEM) [6], la Société française de médecine d'urgence (SFMU) souhaite exprimer ses préoccupations sur les points suivants :

- le choix du triage comme point de départ de la prise en charge réduit le délai pour l'identification et le traitement des patients présentant un sepsis. Dans des structures d'urgences parfois surchargées, la probabilité de réaliser ce protocole de prise en charge en une heure est faible; en particulier, pour les patients qui ne sont pas en état de choc. Les auteurs de la SSC le reconnaissent. Cependant, en médecine d'urgence, imposer des objectifs non réalisables comme indicateurs qualité peut engendrer des dommages inattendus $[7,8]$ et avoir des conséquences médicolégales. Cette diminution du délai, combinée à des critères d'inclusion peu sensibles ou spécifiques (un qSOFA [Quick Sequential Organ Failure Assessment] au moins égal à 2 ou un épisode d'hypotension ou encore une simple valeur de lactate élevée), pourrait exposer les patients à des antibiothérapies et des remplissages inutiles, voire délétères. L'indication du remplissage vasculaire se pose sur les critères d'hypotension ou d'hyperlactatémie ; ainsi, 
des patients présentant une décompensation cardiaque peuvent être exposés à un remplissage vasculaire potentiellement nocif pour eux. De même, un lactate initial isolé élevé est peu spécifique du diagnostic de sepsis, en l'absence d'autres éléments évocateurs. Ainsi, il est rapporté que près d'un patient sur cinq diagnostiqué avec un sepsis aux urgences est, au final, non infecté [9];

- nous regrettons l'utilisation répétée du terme « démontrer» dans la mise à jour de 2018. En effet, la plupart des études citées dans cette nouvelle version décrivent uniquement une association entre respect des recommandations et amélioration du pronostic plutôt qu'un lien de causalité. De plus, d'autres études n'ont pas confirmé cette association $[10,11]$. Nous pensons que les niveaux de preuve conduisant à réduire les délais à une heure sont trop faibles pour être si fortement recommandés. Seymour et al. ont démontré que seuls le prélèvement rapide des hémocultures, du lactate et l'administration précoce d'antibiotiques à large spectre étaient corrélés à la mortalité hospitalière alors que le remplissage vasculaire ne l'était pas [5]. Par ailleurs, après la publication de la troisième définition internationale du sepsis (SEP-3) et parce que le score SOFA (Sequential Organ Failure Assessment) est rarement disponible dans l'heure suivant le triage, la SSC permet d'identifier les patients avec un score qSOFA supérieur ou égal à 2 comme à risque élevé de mortalité [12]. Ainsi, il n'est pas clairement précisé si le qSOFA supérieur ou égal à 2 doit être vu comme un critère de déclenchement du sepsis bundle. Se reposer sur le score SOFA rendrait la recommandation impossible à suivre, car celui-ci nécessite plus d'une heure pour être calculé, ce qui laisse le lecteur dans l'incertitude sur les critères d'initiation du sepsis bundle. Ajoutant à la confusion, la SSC précise dans une réponse à la parution des dernières définitions dites SEPSIS-3 que ce qSOFA ne définit pas l'état de sepsis, sans clairement trancher sur l'indication ou non d'initiation du sepsis bundle. De plus, dans certaines études, le lactate n'apporte pas de valeur pronostique supplémentaire aux scores SOFA ou qSOFA. D'une part, l'exigence d'une mesure répétée du lactate si la première est supérieure à $2 \mathrm{mmol} / \mathrm{l}$ est fondée sur un faible niveau de preuve [13]. D'autre part, Lemachatti et al. suggèrent que la mesure répétée du qSOFA apporte autant d'information que celle du lactate [14]. Nous concluons donc que l'élément clé de la prise en charge est l'administration précoce d'antibiotiques, dès lors que le diagnostic de sepsis devient le plus probable [5] ;

- l'utilisation d'indicateurs fondés sur les délais de prise en charge est une mesure indirecte courante de la qualité des soins réalisés. Il peut même y avoir des incitations financières associées à ces indicateurs. Les recommandations de la SSC pourraient être utilisées par les tutelles ou les régulateurs pour inciter les soignants à respecter cet objec- tif d'une heure devenu indicateur. Cet objectif, qui exigerait que tous les patients présentant un sepsis reçoivent un protocole de prise en charge dans l'heure suivant leur triage dans une structure des urgences, a peu de chances d'être atteint, car le sepsis peut difficilement être diagnostiqué dans ce délai pour des raisons médicales.

$\mathrm{Au}$ final, nous reconnaissons que le sepsis est une pathologie fréquente et associée à une mortalité importante. Les urgentistes sont en première ligne et diagnostiquent un tiers des cas de sepsis [15], les deux autres tiers étant diagnostiqués en salle ou en réanimation. Nous convenons que la rédaction des protocoles de prise en charge doit contribuer à prendre en charge précocement ces patients atteints d'un sepsis, mais les recommandations doivent être fondées sur des données solides. Avoir pour objectif de réaliser le protocole de prise en charge préconisé par la SSC dans l'heure qui suit le triage n'est pas fondé sur des preuves scientifiques suffisamment solides, et cet objectif peut même être délétère pour le patient. À l'instar de l'EUSEM, la SFMU met l'accent sur le dépistage et le traitement précoce du sepsis, sans pour autant valider ces nouvelles recommandations de la SSC. L'association entre antibiothérapie tardive et augmentation de la mortalité ne fait pas débat. La priorité doit être donnée à l'amélioration du diagnostic précoce pour une prise en charge plus adaptée et in fine une amélioration du pronostic.

Liens d'intérêts : les déclarent ne pas avoir de liens d'intérêts.

\section{Références}

1. Levy MM, Evans LE, Rhodes A (2018) The Surviving Sepsis Campaign bundle: 2018 update. Crit Care Med 46:997-1000

2. Rhodes A, Evans LE, Alhazzani W, et al (2016) Surviving Sepsis Campaign: international guidelines for management of sepsis and septic shock. Crit Care Med 45:486-552

3. Leisman DE, Doerfler ME, Ward MF, et al (2017) Survival benefit and cost savings from compliance with a simplified 3-hour sepsis bundle in a series of prospective, multisite, observational cohorts. Crit Care Med 45:395-406

4. Liu VX, Morehouse JW, Marelich GP, et al (2016) Multicenter implementation of a treatment bundle for patients with sepsis and intermediate lactate values. Am J Respir Crit Care Med 193:1264-70

5. Seymour CW, Gesten F, Prescott HC, et al (2017) Time to treatment and mortality during mandated emergency care for sepsis. N Engl J Med 376:2235-44

6. Freund Y, Khoury A, Mockel M, et al (2019) European Society of Emergency Medicine position paper on the one-hour sepsis bundle of the Surviving Sepsis Campaign: expression of concern. Eur J Emerg Med (in press)

7. Mason S, Weber EJ, Coster J, et al (2012) Time patients spend in the emergency department: england's 4-hour rule, a case of hitting the target but missing the point? Ann Emerg Med 59:341-9 
8. Locker TE, Mason SM (2005) Analysis of the distribution of time that patients spend in emergency departments. BMJ 330:1188-9

9. Jones AE, Heffner AC, Horton JM, Marchick MR (2010) Etiology of Illness in patients with severe sepsis admitted to the hospital from the emergency department. Clin Infect Dis 50:814-20

10. Rhee C, Filbin MR, Massaro AF, et al (2018) Compliance with the national Sep-1 quality measure and association with sepsis outcomes: a multicenter retrospective cohort study. Crit Care Med 46:1585-91

11. Finfer S (2010) The Surviving Sepsis Campaign: robust evaluation and high-quality primary research is still needed. Intensive Care Med 36:187-9
12. Antonelli M, Debacker D, Dorman T, et al (2019) Surviving sepsis campaign responds to Sepsis-3. http:/www.survivingsepsis.org/SiteCollectionDocuments/SSC-Statements-Sepsis-Definitions-3-2016.pdf (Dernier accès le 14 mars 2019)

13. Gu WJ, Zhang Z, Bakker J (2015) Early lactate clearance-guided therapy in patients with sepsis: a meta-analysis with trial sequential analysis of randomized controlled trials. Intensive Care Med 41:1862-3

14. Lemachatti N, Ortega M, Penaloza A, et al (2019) Early variation of quick sequential organ failure assessment score to predict inhospital mortality in emergency department patients with suspected infection. Eur J Emerg Med (in press)

15. Lo R, Brabrand M, Kurland L, et al (2019) Sepsis, where are the emergency physicians? Eur J Emerg Med 23:159 